\title{
Differential Fly-Throughs (DFT): A General Framework for Computing Flight Paths
}

\author{
M. Sabry Hassouna ${ }^{1}$, Aly A. Farag ${ }^{1}$, and Robert Falk ${ }^{2}$ \\ 1 Computer Vision \& Image Processing Laboratory (CVIP), \\ University of Louisville, Louisville, Kentucky, 40292 \\ \{msabry, farag\}@cvip.uofl.edu \\ 2 Director of medical imaging, Jewish Hospital, Louisville, Kentucky \\ robert.falk@jhhs.org
}

\begin{abstract}
In this paper, we propose a new variational framework based on distance transform and gradient vector flow, to compute flight paths through tubular and non-tubular structures, for virtual endoscopy. The proposed framework propagates two wave fronts of different speeds from a point source voxel, which belongs to the medial curves of the anatomical structure. The first wave traverses the $3 \mathrm{D}$ structure with a moderate speed that is a function of the distance field to extract its topology, while the second wave propagates with a higher speed that is a function of the magnitude of the gradient vector flow to extract the flight paths. The motion of the fronts are governed by a nonlinear partial equation, whose solution is computed efficiently using the higher accuracy fast marching level set method (HAFMM). The framework is robust, fully automatic, and computes flight paths that are centered, connected, thin, and less sensitive to boundary noise. We have validated the robustness of the proposed method both quantitatively and qualitatively against synthetic and clinical datasets.
\end{abstract}

\section{Introduction}

Virtual endoscopy (VE) is a computer-based alternative to true fiber optic endoscopy (TE) for screening hollow organs. VE is not intended to replace TE, but rather to complement it by providing additional supportive information. For example VE: (1) allows the visualization of neighboring structures outside the screened organ, and hence can assist in the pathology localization, (2) allows viewing in forward and reverse directions, (3) visualize areas that are hard to reach by TE, (4) has the ability to pass high grade stenoses, and finally (5) $\mathrm{VE}$ is the only alternative offered to those patients that either refuse TE or are severely ill [1,2].

The extraction of $3 \mathrm{D}$ flight paths or discrete curve skeletons $(\mathcal{C S})$ of anatomical structures [3-10] is an important component of any VE system. We have recently developed a level set based-framework for computing $\mathcal{C S}$ of 3D tubular and articulated objects [11, which addresses several shortcomings of existing techniques. The key idea is to propagate from a medial voxel wave fronts of different speeds. The first front propagates with a moderate speed to capture the 


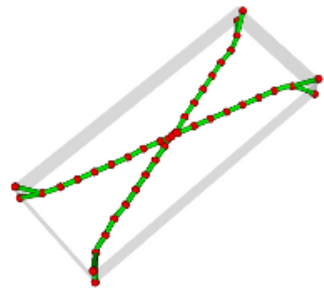

(a)

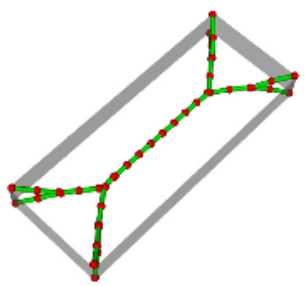

(b)

Fig. 1. Computed $\mathcal{C S}$ for non-tubular structure by (a) [12. (b) Proposed framework.

object topology, while the second one propagates much faster at medial voxels such that $\mathcal{C S}$ intersect the propagating fronts at those voxels of maximum positive curvatures. The framework is slightly modified and quantitatively validated to show that the computed $\mathcal{C S}$ can be used as reliable flight paths through tubular structures for VE 12. In [12, if the structure's cross section deviates too much from the circle, for example, in case of a severe stenoses, where the cross section is nearly rectangular, the computed paths are the shortest as shown in Figure 1(a) rather than the centered as shown in Figure 1(b), which is generated by the proposed framework of this paper.

In this paper, we extend our recent framework [12 to compute flight paths for both tubular and non-tubular structures by utilizing the magnitude of the gradient vector flow, because it does not form medial surfaces for 3D objects.

\section{Methods}

\subsection{Gradient Vector Flow}

The gradient vector flow $(\mathrm{GVF})$ is a bi-directional external force field that moves active contours in highly concave regions [13]. The GVF is the vector field $V(\mathbf{x})=$ $[u(\mathbf{x}) v(\mathbf{x}) w(\mathbf{x})]^{T}$, which minimizes the following energy function,

$$
E(V)=\iiint \mu|\nabla V(\mathbf{x})|^{2}+|\nabla f(\mathbf{x})|^{2}|V(\mathbf{x})-\nabla f(\mathbf{x})|^{2} d \mathbf{x}
$$

Where $\mathbf{x}=(x, y, z), \mu$ is a regularization parameter, and $f(\mathbf{x})$ is an edge map derived from the imaging volume $I(\mathbf{x})$. For a binary volume, $f(\mathbf{x})=-I(\mathbf{x})$. The interpretation of Eq. (11) is that if $|\nabla f(\mathbf{x})|$ is small, $E(V)$ is dominated by the sum of squares of the partial derivatives of the vector field, yielding a slowly varying field. On the other hand, if $|\nabla f(\mathbf{x})|$ is large, $E(V)$ is dominated by the second term, and is minimized by setting $V=\nabla f(\mathbf{x})$. This produces a vector field $V(\mathbf{x})$ that is nearly equal to the gradient of the edge map $\nabla f(\mathbf{x})$ when it is large and slowly varying in homogeneous regions. $V(\mathbf{x})$ can be computed iteratively by solving the following decoupled partial differential equations in $u$, $v$, and $w$ [13. 


$$
\begin{aligned}
u(\mathbf{x}, t+1) & =u(\mathbf{x}, t)+\Delta t\left(\mu \nabla^{2} u(\mathbf{x}, t)-\left(u(\mathbf{x})-f_{x}(\mathbf{x})\right)|\nabla f(\mathbf{x})|^{2}\right) \\
v(\mathbf{x}, t+1) & =v(\mathbf{x}, t)+\Delta t\left(\mu \nabla^{2} v(\mathbf{x}, t)-\left(v(\mathbf{x})-f_{y}(\mathbf{x})\right)|\nabla f(\mathbf{x})|^{2}\right) \\
w(\mathbf{x}, t+1) & =w(\mathbf{x}, t)+\Delta t\left(\mu \nabla^{2} w(\mathbf{x}, t)-\left(w(\mathbf{x})-f_{z}(\mathbf{x})\right)|\nabla f(\mathbf{x})|^{2}\right) \\
V(x, 0) & =\nabla f(\mathbf{x})
\end{aligned}
$$

The iterative process is guaranteed to converge if

$$
\triangle t \leq \frac{\Delta x \Delta y \Delta z}{6 \mu}
$$

where $\Delta x, \Delta y$, and $\Delta z$ are the data spacing of a given dataset. In [14, Xu has shown that the GVF possesses as well some medial properties by suggesting two different medialness functions based on the magnitude of the GVF, to measure only how close a point from the skeleton of a $2 \mathrm{D}$ shape, rather than computing the skeleton itself. One of the interesting properties of the GVF, $V(\mathbf{x})$ over the distance field $D(\mathbf{x})$ is that it does not form medial surfaces for non-tubular 3D objects [15] because only one boundary voxel contributes to the computation of $D(\mathbf{x})$, while more than one boundary voxels contribute to the computation of $V(\mathbf{x})$ during the diffusion process.

\subsection{Single Flight Path Extraction}

Consider the minimum-cost path problem that finds the path $C(s):[0, \infty) \longrightarrow$ $R^{n}$ that minimizes the cumulative travel cost from a starting point $A$ to some destination $B$ in $R^{n}$. If the cost $U$ is only a function of the location $\mathbf{x}$ in the image domain, the cost function is called isotropic, and the minimum cumulative cost at $\mathbf{x}$ is defined as

$$
T(\mathbf{x})=\min \int_{A}^{B} U(C(s)) d s
$$

The path that gives the minimum integral is the minimum cost path. The solution of Eq. (7) is a nonlinear partial differential equation known as the Eikonal equation[16] Eq. (8), where $F(\mathbf{x})=1 / U(\mathbf{x})$, and $T(\mathbf{x})$ is the time at which the front crosses $\mathbf{x}$.

$$
|\nabla T(\mathbf{x})| F(\mathbf{x})=1.0
$$

In this paper, we solve the Eikonal equation using the higher accuracy fast marching method (HAFMM) [17. Let $A$ and $B$ be medial voxels. Assume that $A$ is a point source $P_{S}$ that transmits a high speed front Eq. (9), where $\lambda(\mathbf{x})$ is a medial descriptor function that distinguishes medial voxels from others and $\alpha$ controls the curvature of the front at medial voxels. In [12, we showed that the minimum cost path between $A$ and $B$ is the medial curve or centerline connecting them. Since the GVF does not form medial surfaces in 3D, we propose the following medial descriptor function Eq. (10), where the magnitude of GVF goes to zero at medial voxels.

$$
F(\mathbf{x})=e^{\alpha \lambda(\mathbf{x})} \alpha \geq 0
$$




$$
\lambda(\mathbf{x})=\frac{2.0}{1.0+\left\|V_{n}(\mathbf{x})\right\|^{0.05}}-1, \quad\left\|V_{n}(\mathbf{x})\right\|=\frac{\|V(\mathbf{x})\|-\|V(\mathbf{x})\|_{\min }}{\|V(\mathbf{x})\|_{\max }-\|V(\mathbf{x})\|_{\min }}
$$

The propagating front is monotonically increasing in time; there is only one global minimum over the cumulative cost field $T$, that is $P_{S}$, which has zero travel time. Then, the path between $B$ and $A$ can be found by backtracking from $B$ along the gradient of $T$ until $A$ is reached. The extraction process is the solution of the ordinary differential equation Eq. (11). $C(t)$ traces out the $\mathcal{C} \mathcal{S}$, which is found by solving Eq.(11) using Runge-Kutta of order 2. The error of the method is $O\left(h^{3}\right)$, where $h$ is the integration step. $h$ is set to 1.0 .

$$
\frac{d C}{d t}=-\frac{\nabla T(\mathbf{x})}{|\nabla T(\mathbf{x})|}, C(0)=B
$$

For $C_{i}=\left[x_{i}, y_{i}, z_{i}\right]^{T}$,

$$
f\left(C_{i}\right)=-\frac{\nabla T\left(C_{i}\right)}{\left\|\nabla T\left(C_{i}\right)\right\|}, \quad k_{1}=h f\left(C_{i}\right), \quad C_{i+1}=C_{i}+h f\left(C_{i}+\frac{k_{1}}{2}\right)
$$

\subsection{Multiple Flight Path Extraction}

In order to extract the entire $\mathcal{C S}$ of an anatomical structure, we have to identify the starting voxel (node) of each $\mathcal{C S}$ as well as the merging voxel, if the structure contains a loop. To achieve the goal, we follow the cluster graph (CG) approach [11, which can be summarized as follows: Initially, we compute the normalized distance field $D(\mathbf{x})$ using the HAFMM. Then, we propagate a moderate speed wave from $P_{S}$, which results in a new distance field $D_{1}(\mathbf{x})$. The speed of the front is given by Eq. (13).

$$
F(\mathbf{x})=e^{\beta D(\mathbf{x})}
$$

$D_{1}(\mathbf{x})$ is discretized by computing its integer values. The Discretization converts the structure into a cluster graph, whose root is the cluster containing $P_{S}$. Each cluster consists of a set of neighbor voxels with the same code. The CG contains two main types of clusters; Extreme cluster (Xcluster), which exists at the tail of the CG and Merging cluster (Mcluster), which exists if the structure contains a loop. The point source $P_{S}$ is any medial voxel that belongs to the $\mathcal{C S}$ of the structure, which is the found by searching the CG for the voxel with maximum $D(\mathbf{x})$. If there exists more than one voxels with the same maximum value, we select the one with minimum $\|V(\mathbf{x})\|$. The medial voxel of a cluster is computed similarly to $P_{S}$ but with searching the cluster rather than the CG.

The proposed framework can be summarized as follows: (1) Construct the gradient vector flow $V(\mathbf{x})$ and then compute $\lambda(\mathbf{x})$ Eq. (10), (2) Construct the minimum distance field $D(\mathbf{x})$, (3) Find the point source $P_{S}$, (4) Propagate a moderate speed wave from $P_{S}$ Eq. (13), discretize the resultant distance field $D_{1}(\mathbf{x})$, and construct the CG, (5) Identify the extreme and merging nodes, (6) Construct a new distance field $D_{2}(\mathbf{x})$ from $P_{S}$ by propagating a fast speed wave Eq. (9), (7) If the object contains loops, extract their $\mathcal{C S}$ as suggested in [12, and finally, (8) Extract those $\mathcal{C S}$ that originate from extreme nodes and ends with $P_{S}$ or ends on a previously extracted path to prevent overlapped paths. 


\section{Results and Discussion}

We have quantitatively validated the proposed framework against ground truth $\mathcal{C S}$ that are generated analytically and then discretized. Each phantom is created by translating a sphere of a fixed or varying radius along its ground truth $\mathcal{C} \mathcal{S}$. The phantoms are designed to mimic the geometrical and topological properties of anatomical structures such as: (1) high curvature and torsion (e.g., blood vessels), (2) sudden change in the organ's cross section (e.g., colon or aneurysm in vessels), and (3) several branching nodes (e.g., blood vessels and tracheobronchial trees). To study the sensitivity of the proposed method to noise, $50 \%$ of phantom's boundary voxels are corrupted by additive noise to simulate segmentation error as shown in Figure 2, A quantitative analysis was carried out by computing the amount of overlap, average, and maximum distance between the ground truth and computed $\mathcal{C S}$ for both noise-free and noisy phantoms. The quantitative results are presented in Table 1 . Although the amount of overlap is less than $90 \%$, the average and maximum distance never exceeded 0.42 and 1.41 $\mathrm{mm}$ (e.g., 1-2 voxels), respectively. In the presence of noise, the amount of overlap has been decreased by only $8 \%$, while the average and maximum distance has been increased slightly. To conclude, the computed $\mathcal{C S}$ are always adjacent to the ground truth ones, which is quite acceptable for flight paths in VE.

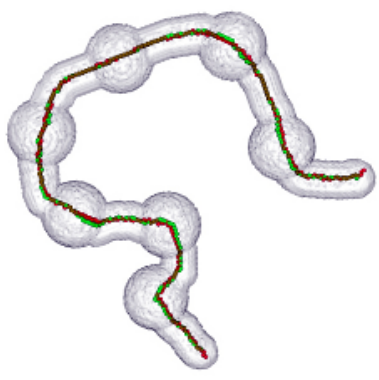

(a) Colon

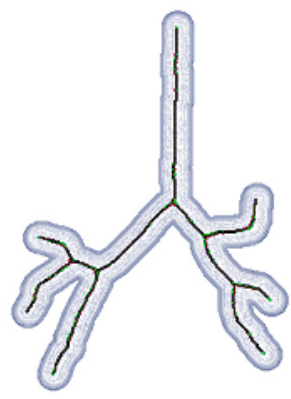

(b) Trachea

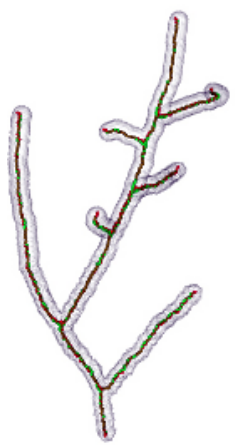

(c) Vessels

Fig. 2. Noisy synthetic phantoms: (a) Colon. (b) Trachea. (c) Vessels. The voxels of the ground truth, computed, and overlapped $\mathcal{C S}$ are represented by green, red, and black spheres, respectively.

Table 1. Quantitative validation for noise-free and noisy phantoms

\begin{tabular}{c|c|c|c|c|c|c}
\hline Phantom & \multicolumn{2}{|c|}{ Colon } & \multicolumn{2}{c|}{ Trachea } & \multicolumn{2}{c}{ Vessels } \\
\hline Size & $200 \times 357 \times 50$ & $200 \times 357 \times 50$ & $220 \times 110 \times 210$ \\
\hline State & Noise-Free & Noisy & Noise-Free & Noisy & Noise-Free & Noisy \\
\hline Percentage of Overlap & $72 \%$ & $65 \%$ & $82 \%$ & $76 \%$ & $65 \%$ & $64 \%$ \\
\hline Average Distance $(\mathrm{mm})$ & 0.33 & 0.42 & 0.18 & 0.19 & 0.42 & 0.46 \\
\hline Maximum Distance $(\mathrm{mm})$ & 1.41 & 2.0 & 1.0 & 1.41 & 1.41 & 2.0 \\
\hline
\end{tabular}


Case Study (Virtual Colonoscopy): The goal of this case study is to bring about one of the important goals of virtual colonoscopy (VC) as a diagnostic tool. The ideal scenario is that the segmented colon maintains the intricate details of the real colon, and that the virtual camera projects views as detailed as those shown in real colonoscopy. If that is achieved, then analysis of projected views can be used for automatic colon scanning against abnormalities, and hence an early detection of colon cancer using VC would be a strong possibility. Our research group is currently pursuing this goal.

In order to illustrate the potential of this research in colonoscopy, the proposed framework has been tested on several CT datasets acquired using Siemens Sensation CT scanner. The dataset volume is $512 \times 512 \times 580$ with voxel size $0.74 \times$ $0.74 \times 0.75$. All patients have undergone standard cleansing preparations prior to scan. In Figure3(e-1), we show different polyps captured by the virtual camera for different colons as shown in Figure 3(a-d). The average running time for these large datasets was 9 minutes on a 2.6GHz AMD Linux workstation with 4.0 GB RAM.

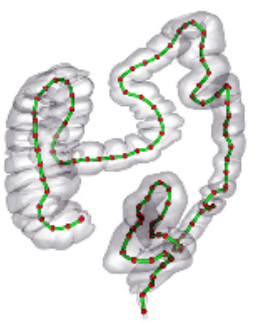

(a) Colon 1

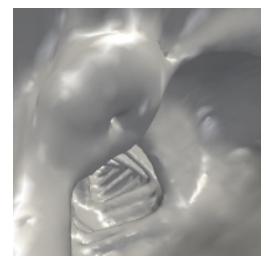

(e)

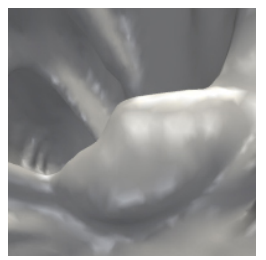

(i)

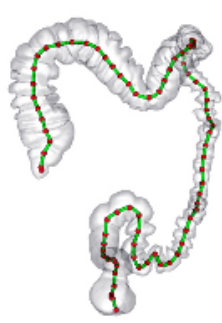

(b) Colon 2

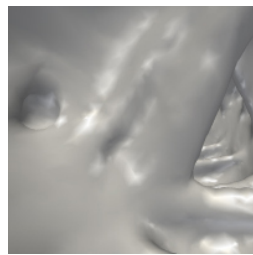

(f)

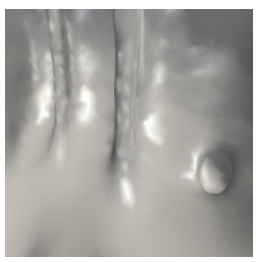

(j)

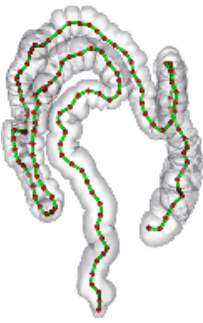

(c) Colon 3

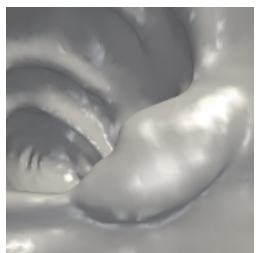

(g)

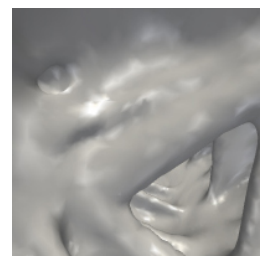

(k)

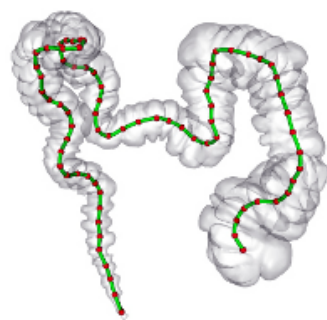

(d) Colon 4

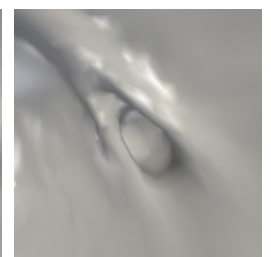

(h)

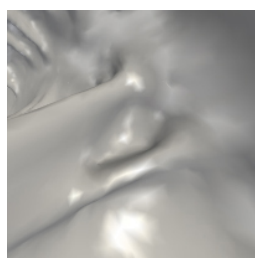

(1)

Fig. 3. Virtual Colonoscopy (a-d) Computed $\mathcal{C S}$ for different colon datasets (e-l) Polyp views captured by the virtual camera 
The proposed framework is controlled by two main parameters $\alpha$ in Eq.(10), which controls the centeredness of the computed flight path and $\beta$ in Eq. (13), which controls the generation of the CG. Experimental results showed that the lower bound for $\alpha$ is equal to 15.0 , while a suitable range of $\beta$ is $2.0<\beta<4.0$. We automated the framework by setting $\alpha=20.0$ and $\beta=3.0$.

We have slightly modified the original GVF to suit the nature of the problem as follows: (1) $f(\mathbf{x})=I(\mathbf{x})$ such that the vector field points towards the center of the object. (2) The computation of the GVF is restricted to the internal voxels of the structure and hence computationally more efficient than the original GVF. (3) The GVF field is not normalized to maintain the medialness property, otherwise the magnitude of the GVF is unity everywhere. The GVF parameters are set as follows: $\mu=0.15, \Delta t=0.5$, and the number of iterations is set to 100 .

The complexity of the framework in the worst case for $n$ voxels is $O(3 n \log n+$ $k n$ ). The complexity of computing the distance field using the HAFMM and the GVF is given by nlogn and $k n$, respectively, where $k$ is the number of iterations.

\section{Conclusion and Future Work}

In this paper, we have proposed a general framework for computing flight paths through tubular and non-tubular structures using partial differential equations (PDE). Unlike all voxel-based methods, the framework does not require voxel sizes to be isotropic because the solution of the PDE takes into account the data spacing. The computed flight paths are highly centered, connected, topology preserving, and forms a graph at no additional cost.

Most of the processing time of the proposed method is spent on the computation of the GVF because it requires extensive floating point operations. Therefore, in the future, we intend to implement the GVF in the graphical processing unit (GPU) to alleviate its computational complexity.

Acknowledgment. The colonoscopy dataset used in this paper were provided by 3DR Inc., Louisville, Kentucky.

\section{References}

1. Baert, A.L., Sartor, K.: Virtual Endoscopy and Related 3D Techniques. Springer (2001)

2. Buthiau, D., Khayat, D.: Virtual Endoscopy. Springer-Verlag (2003)

3. Zhou, Y., Toga, A.W.: Efficient skeletonization of volumetric objects. IEEE Transactions on Visualization and Computer Graphics 5 (1999) 196-209

4. Bitter, I., Kaufman, A.E., Sato, M.: Penalized-distance volumetric skeleton algorithm. IEEE Transactions on Visualization and Computer Graphics 7 (2001) 195-206

5. Ma, C., Sonka, M.: A fully parallel 3d thinning algorithm and its applications. Computer Vision and Image Understanding 64 (1996) 420-433

6. Svensson, S., Nyström, I., di Baja, G.S.: Curve skeletonization of surface-like objects in 3d images guided by voxel classification. Pattern Recognition Letters 23 (2002) 1419-1426 
7. Deschamps, T.: Curve and Shape Extraction with Minimal Path and Level-Sets techniques - Applications to 3D Medical Imaging. PhD thesis, Université Paris-IX Dauphine (2001)

8. Bouix, S., Siddiqi, K., Tannenbaum, A.: Flux driven fly throughs. In: Computer Vision and Pattern Recognition, CVPR. (2003) 449-454

9. Aylward, S.R., Bullitt, E.: Initialization, noise, singularities, and scale in height ridge traversal for tubular object centerline extraction. IEEE Trans. Medical Imaging 21 (2002) 61-75

10. Wink, O., Niessen, W., Viergever, M.: Multiscale vessel tracking. IEEE Trans. Medical Imaging 23 (2004) 130-133

11. Hassouna, M.S., Farag, A.A.: Robust centerline extraction framework using level sets. In: Proc. of IEEE Conference on Computer Vision and Pattern Recognition, CVPR, San Diego, CA (2005) 458-465

12. Hassouna, M.S., Farag, A.A.: Pde-based three dimensional path planning for virtual endoscopy. In: Proc. of Information Processing in Medical Imaging, IPMI, Glenwood springs, Colorado (2005)

13. Xu, C., Prince, J.L.: Gradient vector flow: A new external force for snakes. In: CVPR '97: Proceedings of the 1997 Conference on Computer Vision and Pattern Recognition (CVPR '97), Washington, DC, USA (1997) 66-71

14. Xu, C.: Deformable Models with Application to Human Cerebral Cortex Reconstruction from Magnetic Resonance Images. PhD thesis, Department of Electrical and Computer Engineering, Johns Hopkins University (1999)

15. Chang, S., Metaxas, D., Axel, L.: Scan-conversion algorithm for ridge point detection on tubular objects. In: MICCAI, MontrTal, Canada (2003) 158-165

16. Cohen, L., Kimmel, R.: Global minimum for active contour models: A minimal path approach. International Journal of Computer Vision 24 (1997) 57-78

17. Sethian, J.: Level Sets Methods and Fast Marching Methods. 2nd edn. Cambridge University Press (1999) 